\title{
Management of Eastern Brown and Tiger Snake Envenomation in Domestic Animals in South Australia
}

\author{
Madeleine J. Wright ${ }^{1}$ and Suong N. T. Ngo ${ }^{1 *}$ \\ ${ }^{1}$ School of Animal and Veterinary Sciences, The University of Adelaide, Roseworthy, SA 5371, \\ Australia.
}

Authors' contributions

This work was carried out in collaboration between both authors. Author MJW performed the collection and analysis of the data. Author SNTN designed the study, managed the analyses and interpretation of the data, and prepared the manuscript. Both authors read and approved the final

manuscript.

Article Information

DOI: $10.9734 / A R R B / 2018 / 40513$

Editor(s):

(1) George Perry, Dean and Professor of Biology, University of Texas at San Antonio, USA.

Reviewers:

(1) Khin Than Yee, Myanmar.

(2) Jaya Vejayan, Universiti Malaysia Pahang, Malaysia.

(3) F. A. Adamude, Federal University Lafia, Nigeria.

Complete Peer review History: http://www.sciencedomain.org/review-history/24024

Original Research Article

Received $24^{\text {th }}$ January 2018

Accepted $30^{\text {th }}$ March 2018

Published 6 ${ }^{\text {th }}$ April 2018

\section{ABSTRACT}

Aims: The aims of this study were to examine the key important clinical features in domestic animals (e.g. dogs and cats) accompanying envenomation by eastern brown or tiger snakes in South Australia, to establish whether routine use of snake venom detection kits (SVDKs) is a useful diagnostic approach for identifying offending snake species and help to improve animal's condition and survival.

Methodology: Clinical records from 155 dogs and cats, with suspected or confirmed snake envenomation were collected from two veterinary practices in South Australia. Chi-square analysis was used to compare patient outcome with diagnostic method, and descriptive analysis were undertaken to compare features between the envenomation cases.

Results: Brown snakes were responsible for $97 \%$ of cases, with the remaining $3 \%$ due to tiger snakes. The most common presenting signs were recumbency and hind limb paresis. Patients were 
$20 \%$ less likely to survive when diagnosis of the offending snake species was made by means other than the use of a SVDK. An inconclusive result occurred approximately $14 \%$ of the time when using a SVDK. The overall survival rate for cases that were treated was $81 \%$ in dogs and $92 \%$ in cats.

Conclusion: Species-specific snake diagnosis using a SVDK will lead to improved patient outcome, however care is required when performing and interpreting the test due to a high proportion of contradictory results obtained.

Keywords: Snake envenomation; snakebite; antivenom; domestic animals; dogs; cats.

\section{INTRODUCTION}

The incidence of snake envenomation is quite common in domestic animals, especially in rural areas of Australia. A study by Mirtschin et al. [1] had estimated that approximately 6240 confirmed or suspected cases of snakebite occur in domestic animals annually, however the actual value is likely to be substantially higher. This was due to the fact that animals bitten by snakes may not always be brought to veterinary clinics for treatment. In South Australia, the venomous snakes found most frequently in residential areas include the following species: Eastern brown snake (Pseudonaja textilis), red-bellied black snake (Pseudechis porphyriacus), copperhead snake (Austrelaps superbus), western brown snake (Pseudonaja nuchalis), and tiger snake (Notechis scutatus) [2]. The vast majority of envenomations seen in South Australia however are due to the eastern brown snake [3]. Current methods used by veterinary clinicians to diagnose snake envenomation in domestic animals include clinical signs, laboratory tests (haematology, biochemistry, coagulation profile), snake venom detection kits (SVDKs), snake identification and geographical knowledge of species common to the area [3]. Diagnosis based on clinical signs alone has been proven unreliable due to the significant overlap of envenomation syndromes between snake species [4]. A study by Morrison and co-workers [5] had concluded that only $19 \%$ of common Australian snake species could be identified by individuals of a range of ages and demographics, highlighting the importance of using a combination of methods in order to achieve an accurate diagnosis.

The CSL SVDK is a rapid ELISA immunoassay, which is used to detect venom from snakes in Australia and Papua New Guinea [6]. It is currently used infrequently in the majority of veterinary practices, with a survey by Mirtschin and colleagues [1] reporting their use in only $1 \%$ of cases. The reasoning behind this includes expense, excessive running time and perceived inaccuracy of the test [7]. There have been numerous reports of contradictory results being obtained from the SVDK, with majority of problems likely arising due to operator error [8] or inappropriate sample selection based on time since envenomation [9]. A positive result without any other evidence of snakebite does not always indicate that clinical envenomation has occurred. Likewise, a negative result in patients showing clinical signs of envenomation is not a reason for withholding antivenom [6].

Whilst there has been some research that investigated the effectiveness of the SVDK in accurately detecting snake envenomation $[3,8,9,10-14]$, limited work has been done to compare the specific diagnostic methods and to relate them to survival of the treated animal. A recent study describing clinical features of tiger and brown snake envenomation in dogs and cats was performed in Victoria. However its limitation was that a total of only 5 brown snakebites were confirmed, making it difficult to describe and compare the envenomation syndromes [15]. The aims of the present study were to determine if patient outcome was influenced by the method(s) used to diagnose snake species, to establish if it is justifiable for veterinary clinics to use SVDKs routinely, and to highlight the clinical features associated with eastern brown and tiger snake envenomation in dogs and cats in South Australia.

\section{MATERIALS AND METHODS}

\subsection{Study Design Overview and Data Collection}

A retrospective study, which involved collection of case series, was conducted using records from dogs and cats seeking treatment for suspected snake envenomation from October 2012 to August 2015 at the Companion Animal Health Centre, Roseworthy, and from October 2014 to March 2015 at the Riverland Veterinary Practice, Berri, South Australia. IDEXX Cornerstone ${ }^{\circledR}$ Practice Management Software, 
the data management software used at the two veterinary practices, was used to search for the invoice codes: snake detection kit, brown snake antivenom, and tiger/brown antivenom. Strict confidentiality was adhered to when accessing the data, with all owner information de-identified. The clinical records were transferred from Cornerstone ${ }^{\circledR}$ into Microsoft Excel for analyses.

Data collected from each case included patient ID, species (canine/feline), breed, presenting clinical signs, whether a SVDK was used (yes/no), sample used for SVDK (blood/urine) and result from the SVDK, other diagnostic tests performed, treatment given, clinical outcome, and number of days hospitalised.

\subsection{Group Classification}

Animals were classified as suffering from snake envenomation based on one or more of the following criteria:

1. The animal was seen interacting with a snake.

2. Clinical signs typical of snake envenomation, which include generalised weakness or recumbency, ataxia or hind limb paresis, hypothermia, reduced gag reflex, dyspnoea, mydriasis, and ptyalism.

3. A positive SVDK result.

4. Prolonged coagulation parameters (PT or aPTT) observed.

For the purposes of this study, animals were defined as being definitively diagnosed with brown or tiger snake envenomation based on a positive SVDK, or examination and identification of the snake's scales if it was brought into the clinic. Cases showing clinical signs consistent with snake envenomation, or cases in which animals were seen to interact with a snake but with no definitive diagnosis were characterised as suspected brown or tiger snake bites. Outcome was classified as whether the treated animal survived, died (due to treatment failure), or was euthanased (due to financial restrictions or poor prognosis).

\subsection{Statistical Analysis}

Statistical analyses were performed using Stata IC/13.1 (StataCorp, College Station, TX, USA, 2009) and Statulator (beta). Patient outcome was compared to the method of diagnosing snake envenomation, specifically by use of a SVDK or based on a combination of presenting clinical signs and other diagnostic methods, using Chisquare analysis at a significance level of $P \leq .05$. Evaluation of the percentage of inconclusive or contradictory results provided by the SVDK was assessed for all cases in which a SVDK was used in the diagnostic process.

Descriptive analysis of confirmed or suspected snake envenomation cases was performed for both dogs and cats. Animals that were euthanased due to poor prognosis or died during treatment were also included in the analysis. However, patients that were euthanased for financial reasons were excluded from calculations of survival and mortality rates.

\section{RESULTS}

\subsection{Case Overview}

Based on the search parameters, a total of 155 records were obtained. These cases were further reviewed, and a total of 112 snakebite cases comprising 36 dogs (32\%) and 76 cats $(68 \%)$ were included in the descriptive analysis of snake envenomation. It was noted that 2 cats were presented a total of 3 times, 3 cats presented on 2 separate occasions, and 1 dog brought in 2 times for suspected snake envenomation. There were 2 dogs and 1 cat excluded from the analysis of patient outcome based on diagnostic method due to being euthanased for financial reasons, bringing the total number of cases down to 109. There were 106 suspected snakebite cases in which a SVDK was used, and only 62 of these 106 cases were diagnosed as confirmed snake envenomations. Descriptive statistics, including clinical findings, treatments and outcome are tabulated for both dogs (Table 1) and cats (Table 2).

\subsection{Snake Characteristics}

It is estimated that brown snakes were responsible for a total of 109 cases of snake envenomation (97\%), and tiger snakes only 3 cases (3\%). The majority of dogs (Table 1) and less than one fourth of cats (Table 2) were considered to have interacted with a snake, based on the following observations: finding a dead snake nearby, seen playing with a snake, or seen being bitten by a snake. The bite site was found in only a few dogs, either on the hind limb or the inner surface of the lower lip. No bite sites were found in any cat cases. 
Table 1. Findings in dogs with snake envenomation

\begin{tabular}{|c|c|c|c|c|c|c|}
\hline \multicolumn{7}{|c|}{ Envenomation group } \\
\hline & $\begin{array}{l}\text { Brown } \\
\text { snake }\end{array}$ & $\begin{array}{l}\text { Suspected } \\
\text { brown snake }\end{array}$ & $\begin{array}{l}\text { Tiger } \\
\text { snake }\end{array}$ & $\begin{array}{l}\text { Suspected } \\
\text { tiger snake }\end{array}$ & \multicolumn{2}{|l|}{ Total } \\
\hline Number of dogs & 15 & 19 & 1 & 1 & \multicolumn{2}{|l|}{36} \\
\hline $\begin{array}{l}\text { Seen interacting with the } \\
\text { snake }\end{array}$ & $10(67 \%)$ & $14(74 \%)$ & $1(100 \%)$ & 0 & \multicolumn{2}{|c|}{$25(69 \%)$} \\
\hline $\begin{array}{l}\text { History of collapse and } \\
\text { recovery }\end{array}$ & $4(27 \%)$ & $4(21 \%)$ & 0 & 0 & \multicolumn{2}{|c|}{$8(22 \%)$} \\
\hline Bite site found & $2(13 \%)$ & $1(5 \%)$ & 0 & 0 & \multicolumn{2}{|l|}{$3(8 \%)$} \\
\hline $\begin{array}{l}\text { LAB: Prolonged PT } \\
\text { Prolonged a PTT } \\
\text { Elevated CK }\end{array}$ & & & & & $\begin{array}{l}1 / 15 \\
8 / 12 \\
3 / 9\end{array}$ & $\begin{array}{l}(73 \%) \\
(67 \%) \\
(33 \%)\end{array}$ \\
\hline $\begin{array}{l}\text { SVKD results } \\
\text { Clinical signs: } \\
\text { Neurotoxicity }\end{array}$ & + & - & + & - & & \\
\hline Mydriasis & $1(7 \%)$ & $4(21 \%)$ & 0 & 0 & \multicolumn{2}{|c|}{$5(14 \%)$} \\
\hline Ataxia/hind limb paresis & $3(20 \%)$ & $9(47 \%)$ & 0 & $1(100 \%)$ & \multicolumn{2}{|c|}{$13(36 \%)$} \\
\hline Weakness/recumbency & $5(33 \%)$ & $8(42 \%)$ & 0 & 0 & \multirow{2}{*}{\multicolumn{2}{|c|}{$13(36 \%)$}} \\
\hline Generalised tremors & $2(13 \%)$ & $2(11 \%)$ & 0 & 0 & $4(11 \%)$ & \\
\hline Reduced gag reflex & 0 & $3(16 \%)$ & 0 & 0 & \multicolumn{2}{|l|}{$3(8 \%)$} \\
\hline Dyspnoea & $6(40 \%)$ & $7(37 \%)$ & 0 & $1(100 \%)$ & \multicolumn{2}{|c|}{$14(39 \%)$} \\
\hline Alimentary signs & & & & & & \\
\hline Diarrhoea & $1(7 \%)$ & 0 & 0 & 0 & \multicolumn{2}{|l|}{$1(3 \%)$} \\
\hline Ptyalism & $4(27 \%)$ & $6(32 \%)$ & 0 & 0 & \multicolumn{2}{|c|}{$10(28 \%)$} \\
\hline Vomiting & $2(13 \%)$ & $3(16 \%)$ & & & \multicolumn{2}{|c|}{$5(14 \%)$} \\
\hline Abdominal pain & $1(7 \%)$ & 0 & 0 & 0 & \multicolumn{2}{|c|}{$1(3 \%)$} \\
\hline Other effects & & & & & & \\
\hline Hypothermia $\left(<37.5^{\circ} \mathrm{C}\right)$ & $1(7 \%)$ & $3(16 \%)$ & 0 & 0 & \multicolumn{2}{|c|}{$4(11 \%)$} \\
\hline Pyrexia $\left(>39.2^{\circ} \mathrm{C}\right)$ & $213 \%)$ & 0 & 0 & 0 & \multicolumn{2}{|c|}{$2(6 \%)$} \\
\hline Prolonged CRT (>2sec) & $1(7 \%)$ & 0 & 0 & 0 & \multicolumn{2}{|l|}{$1(3 \%)$} \\
\hline $\begin{array}{l}\text { Pale mucous } \\
\text { membranes }\end{array}$ & $1(7 \%)$ & 0 & 0 & $1(100 \%)$ & \multicolumn{2}{|c|}{$2(6 \%)$} \\
\hline $\begin{array}{l}\text { Injected mucous } \\
\text { membranes }\end{array}$ & $1(7 \%)$ & $6(32 \%)$ & 0 & 0 & \multicolumn{2}{|c|}{$7(19 \%)$} \\
\hline Cyanosis & $1(7 \%)$ & $4(21 \%)$ & 0 & 0 & \multicolumn{2}{|c|}{$5(14 \%)$} \\
\hline Tachycardia & $3(20 \%)$ & $3(16 \%)$ & 0 & $1(100 \%)$ & \multicolumn{2}{|c|}{$7(19 \%)$} \\
\hline Tachypnoea & $1(7 \%)$ & $1(5 \%)$ & 0 & 0 & \multicolumn{2}{|c|}{$2(6 \%)$} \\
\hline Lung crepitation & 0 & $1(5 \%)$ & 0 & 0 & $1(3 \%)$ & \\
\hline Distressed/agitated & $2(13 \%)$ & $2(11 \%)$ & 0 & 0 & $4(11 \%$ & \\
\hline Subdued/lethargic & $2(13 \%)$ & $4(21 \%)$ & 0 & 0 & $6(17 \%$ & \\
\hline Pigmenturia & $1(7 \%)$ & 0 & 0 & 0 & $1(3 \%)$ & \\
\hline Clinical bleeding & $213 \%)$ & $4(21 \%)$ & 0 & 0 & $6(17 \%$ & \\
\hline $\begin{array}{l}\text { Treatment: } \\
\text { Adjunct therapy }\end{array}$ & & & & & & \\
\hline Analgesia & & & & & $13 \%$ & \\
\hline Active warming & & & & & $11 \%$ & \\
\hline AmoxyClav & & & & & $9 \%$ & \\
\hline Furosemide & & & & & $6 \%$ & \\
\hline Urocarb & & & & & $3 \%$ & \\
\hline Atropine & & & & & $3 \%$ & \\
\hline Sedation & & & & & $2 \%$ & \\
\hline Fresh frozen plasma & & & & & $2 \%$ & \\
\hline Anti-emetic & & & & & $2 \%$ & \\
\hline
\end{tabular}




\begin{tabular}{|c|c|c|c|c|c|}
\hline \multicolumn{6}{|c|}{ Envenomation group } \\
\hline & $\begin{array}{l}\text { Brown } \\
\text { snake }\end{array}$ & $\begin{array}{l}\text { Suspected } \\
\text { brown snake }\end{array}$ & $\begin{array}{l}\text { Tiger } \\
\text { snake }\end{array}$ & $\begin{array}{l}\text { Suspected } \\
\text { tiger snake }\end{array}$ & Total \\
\hline \multicolumn{6}{|l|}{ Main therapy } \\
\hline No. of dogs premedicated & $9(60 \%)$ & $15(79 \%)$ & $1(100 \%)$ & 0 & $25(69 \%)$ \\
\hline $\begin{array}{l}\text { Median (range) number } \\
\text { of vials of antivenom } \\
\text { administered }\end{array}$ & $1.9(0-4)$ & $1.6(1-3)$ & 2 & 2 & $1.75(0-4)$ \\
\hline $\begin{array}{l}\text { No. of dogs mechanically } \\
\text { ventilated }\end{array}$ & $2(13 \%)$ & $4(21 \%)$ & 0 & 0 & $6(17 \%)$ \\
\hline $\begin{array}{l}\text { Median (range) number } \\
\text { of days hospitalised }\end{array}$ & $2.25(1-4)$ & $2.5(1-6)$ & 2 & 5 & $2.5(1-6)$ \\
\hline No. of dogs that survived & $12(100 \%)$ & $15(83 \%)$ & $1(100 \%)$ & $1(100 \%)$ & $29(81 \%)$ \\
\hline No. of dogs that died & 0 & $3(17 \%)$ & 0 & 0 & $3(8 \%)$ \\
\hline $\begin{array}{l}\text { No. of dogs euthanased } \\
\text { (financial reasons) }\end{array}$ & $2(13 \%)$ & 0 & 0 & 0 & $2(6 \%)$ \\
\hline $\begin{array}{l}\text { No. of dogs euthanased } \\
\text { (grave prognosis) }\end{array}$ & $1(7 \%)$ & $1(5 \%)$ & 0 & 0 & $2(6 \%)$ \\
\hline
\end{tabular}

\subsection{Clinical Signs}

There were 8 dogs (22\%) that suffered from a history of acute transient collapse with salivation, ataxia and lethargy, followed by spontaneous recovery. Both brown and tiger snake envenomations produced similar clinical syndromes, which were assessed based mainly on empirical approach, with recumbancy (64\%) (59 cats +13 dogs $=72 / 112$ animals $)$ and ataxia/hind limb paresis (52\%) (45 cats +13 dogs $=58 / 112$ animals) considered to be the most common presenting clinical signs. No principal differences were recognised between the species envenomation syndromes, however it is difficult to make any assumptions from this data due to the small number of cases of tiger snake envenomations. As summarised in Tables 1 and 2, specific presenting signs of snakebite observed in dogs included dyspnoea, ptyalism, injected mucous membranes, tachycardia, and lethargy. Whereas in cats the main signs observed included hypothermia, reduced gag reflex, mydriasis, and tachycardia. A small number of dogs and cats were reported to have clinical bleedings, which were manifested as either haemorrhagic diarrhoea, haemorrhage from the bite site, bleeding and haematoma formation at the venepuncture site, or blood coming from the nose and throat. About $70 \%$ of dogs and cats with clinical bleeding survived, with the remaining died or being euthanased due to suspected cerebral haemorrhage.

\subsection{Diagnostic Tests}

Diagnosis based solely on clinical signs, or a combination of clinical signs, prolonged coagulation factors and snake identification was carried out in $44 \%$ of snake envenomation cases, and the other $56 \%$ of the time the diagnosis was made using a SVDK. The findings indicate that dogs and cats were $20 \%$ more likely $(P=0.016)$ to succumb to snake envenomation when diagnosis of the offending snake species was not made using a SVDK. An inconclusive result occurred approximately $14 \%$ of the time when a SVDK was used. This was defined as receiving multiple positives for different snake species, receiving a negative result on the SVDK when all clinical signs and other diagnostic tests suggest snake envenomation, or a positive SVDK result with no clinical signs or diagnostic tests to suspect envenomation. Coagulation tests, specifically prothrombin time (PT) and activated partial thromboplastin time (aPTT) were not performed routinely, thus were only measured for a subset of animals. PT was prolonged in the majority of dogs and in half of cats, whereas, aPTT was prolonged in two third of dogs and also half of cats. For the majority of the dogs described above as having clinical bleeding, they were not tested for coagulation factors, with two dogs had either normal PT or increased aPTT (Tables 1 and 2). For the cats found to have clinical bleeding, coagulation factors were not tested in two cases, with the remaining cats either had increased aPTT, increased PT and aPTT, or normal coagulation parameters. Creatinine kinase (CK) was elevated in one third of dogs, and in about a quarter of cats with suspected or confirmed brown snake envenomation. This measure was not tested in the 2 dogs or cat with suspected or confirmed tiger snake envenomation. 
Table 2. Findings in cats with snake envenomation

\begin{tabular}{|c|c|c|c|c|}
\hline \multicolumn{5}{|c|}{ Envenomation group } \\
\hline & Brown snake & $\begin{array}{l}\text { Suspected } \\
\text { brown snake }\end{array}$ & Tiger snake & Total \\
\hline Number of cats & 37 & 38 & 1 & 76 \\
\hline Seen interacting with the snake & $6(16 \%)$ & $7(18 \%)$ & 0 & $13(17 \%)$ \\
\hline LAB Results: & & & & $12 / 21(57 \%)$ \\
\hline Prolonged PT & & & & $12 / 25(48 \%$ \\
\hline Prolonged a PTT & & & & $4 / 17(24 \%)$ \\
\hline Elevated CK & & & & \\
\hline SVDK results & + & - & + & \\
\hline \multirow{2}{*}{\multicolumn{5}{|c|}{$\begin{array}{l}\text { Clinical Signs: } \\
\text { Neurotoxicity }\end{array}$}} \\
\hline & & & & \\
\hline Mydriasis & $10(27 \%)$ & $14(37 \%)$ & 0 & $24(32 \%)$ \\
\hline Miosis & $2(5 \%)$ & $2(5 \%)$ & 0 & $4(5 \%)$ \\
\hline Reduced pupillary light reflex & $3(8 \%)$ & $4(11 \%)$ & 0 & $7(9 \%)$ \\
\hline Extraocular ophthalmoplegia ${ }^{a}$ & $7(19 \%)$ & $1(3 \%)$ & 0 & $8(11 \%)$ \\
\hline Ataxia/hind limb paresis & $22(59 \%)$ & $22(58 \%)$ & $1(100 \%)$ & $45(59 \%)$ \\
\hline Weakness/recumbency & $30(81 \%)$ & $28(74 \%)$ & $1(100 \%)$ & $59(78 \%)$ \\
\hline Generalised tremors & 0 & $1(3 \%)$ & 0 & $1(1 \%)$ \\
\hline Reduced gag reflex & $12(32 \%)$ & $15(39 \%)$ & $1(100 \%)$ & $28(37 \%)$ \\
\hline Dyspnoea & $6(16 \%)$ & $9(24 \%)$ & 0 & $15(20 \%)$ \\
\hline \multicolumn{5}{|l|}{ Alimentary signs } \\
\hline Diarrhoea & 0 & $2(5 \%)$ & 0 & $2(3 \%)$ \\
\hline Ptyalism & 0 & $4(11 \%)$ & 0 & $4(5 \%)$ \\
\hline Abdominal pain & 0 & $1(3 \%)$ & 0 & $1(1 \%)$ \\
\hline \multicolumn{5}{|l|}{ Other effects } \\
\hline Hypothermia $\left(<38.0^{\circ} \mathrm{C}\right)$ & $20(54 \%)$ & $21(55 \%)$ & $1(100 \%)$ & $42(55 \%)$ \\
\hline Pyrexia $\left(>39.2^{\circ} \mathrm{C}\right)$ & $1(3 \%)$ & 0 & 0 & $1(1 \%)$ \\
\hline Prolonged CRT(>2sec) & $2(5 \%)$ & 0 & 0 & $2(3 \%)$ \\
\hline Pale mucous membranes & $1(3 \%)$ & 0 & 0 & $1(1 \%)$ \\
\hline Injected mucous membranes & 0 & $1(3 \%)$ & 0 & $1(1 \%)$ \\
\hline Cyanosis & $1(3 \%)$ & $1(3 \%)$ & 0 & $2(3 \%)$ \\
\hline Dehydration & $2(5 \%)$ & $1(3 \%)$ & 0 & $3(4 \%)$ \\
\hline Tachycardia & $10(27 \%)$ & $8(21 \%)$ & 0 & $18(24 \%)$ \\
\hline Bradycardia & $3(8 \%)$ & $2(5 \%)$ & & $5(7 \%)$ \\
\hline Tachypnoea & $10(27 \%)$ & $6(16 \%)$ & 0 & $16(21 \%)$ \\
\hline Lung crepitation & $1(3 \%)$ & $1(3 \%)$ & 0 & $2(3 \%)$ \\
\hline Distressed/agitated & $1(3 \%)$ & $4(11 \%)$ & 0 & $5(7 \%)$ \\
\hline Subdued/lethargic & $9(24 \%)$ & $8(21 \%)$ & 0 & $17(22 \%)$ \\
\hline Pigmenturia & 0 & $1(3 \%)$ & 0 & $1(1 \%)$ \\
\hline Clinical bleeding & 0 & $5(13 \%)$ & 0 & $5(7 \%)$ \\
\hline \multicolumn{5}{|l|}{ Treatment: } \\
\hline No. of cats premedicated & $28(76 \%)$ & $29(76 \%)$ & 0 & $57(75 \%)$ \\
\hline $\begin{array}{l}\text { Median (range) number of vials of } \\
\text { antivenom administered }\end{array}$ & $1(0-1.5)$ & $1.1(0-3)$ & $1(1)$ & $1(0-3)$ \\
\hline $\begin{array}{l}\text { No. of cats mechanically } \\
\text { ventilated }\end{array}$ & 0 & $2(5 \%)$ & 0 & $2(3 \%)$ \\
\hline $\begin{array}{l}\text { Median (range) number of days } \\
\text { hospitalised }\end{array}$ & $3.1(1-6)$ & $3.1(1-5)$ & $3(3)$ & $3.1(1-6)$ \\
\hline No. of cats that survived & $37(100 \%)$ & $32(86 \%)$ & $1(100 \%)$ & $70(92 \%)$ \\
\hline No. of cats that died & 0 & $5(14 \%)$ & 0 & $5(7 \%)$ \\
\hline $\begin{array}{l}\text { No. of cats euthanased (financial } \\
\text { reasons) }\end{array}$ & 0 & $1(3 \%)$ & 0 & $1(1 \%)$ \\
\hline
\end{tabular}

${ }^{a}$ Reduced palpebral reflex and reduced menace response 


\subsection{Treatment}

Various supportive drugs and treatments were given to envenomated animals. The most commonly reported treatment options for more than $90 \%$ of snake envenomation were polyvalent antivenom administration and intravenous fluid therapy, with oxygenation required for less than half of the cases. Other treatments that were utilised in a few cases of envenomation included analgesia, active warming, antibiotics, mechanical ventilation, diuretics, urinary antispasmodics, atropine, sedation, fresh frozen plasma, and anti-emetics (See Table 1).

A majority of dogs and cats were premedicated prior to administration of the appropriate antivenom as described in Tables 1 and 2. This included combinations of corticosteroids such as dexamethasone, antihistamines such as niramine, and adrenaline. Brown snake envenomated dogs were given a median of 1.75 vials of polyvalent brown snake antivenom. Of the confirmed dogs in the brown snake envenomation group, one fifth were euthanased due to financial restrictions, and majority of the remaining received an average of 1 vial or 2 vials of polyvalent brown snake antivenom, with a few cases received 3 vials to 4 vials. The two dogs treated for tiger snake envenomation were both given 2 vials of polyvalent tiger/multi-brown snake antivenom (Refer to Table 1 for further details).

Cats were given an average of one vial of brown snake antivenom for brown snake envenomation. Of the confirmed cats in the brown snake envenomation group, one was not given any antivenom due to 2 previous cases of envenomation and antivenom administration, as further antivenom administration may increase the chance of an adverse reaction occurring. The majority of the remaining cats were given either 1 vial of polyvalent brown snake antivenom, with only one animal received 1.5 vials. The cat with tiger snake envenomation was given a vial of polyvalent tiger/multi-brown snake antivenom (See Table 2).

\subsection{Clinical Outcome}

A hypersensitivity reaction following administration of antivenom occurred in $8 \%$ of cats with suspected or confirmed brown snake envenomation. Each of these cats had been premedicated with a combination of corticosteroids and antihistamines. The signs observed with the reactions included dyspnoea, tachypnoea, bradycardia, distress and agitation. No dogs suffered from any hypersensitivity reactions.

The average length of hospitalisation for cases of brown snake envenomation was 2.5 days in dogs and 3.1 days in cats. The hospitalisation period for the dogs with tiger snake envenomation was 2 and 5 days, and the cat with tiger snake envenomation was 3 days.

The survival percentages in dogs that were treated was $100 \%$ in the brown snake envenomation group and for both cases of confirmed and suspected tiger snake envenomation, with $83 \%$ for suspected cases of brown snake envenomation. The survival percentages for cats that were treated was also $100 \%$ in the brown snake envenomation group and the single case of tiger snake envenomation, with $86 \%$ for suspected cases of brown snake envenomation. The overall rate of euthanasia due to financial reasons was $11 \%$ in dogs and $1 \%$ in cats (Refer to Tables 1 and 2).

\section{DISCUSSION}

The majority of envenomations occurred in this study were due to Eastern brown snakes. Brown snakes are reported to be the most common venomous snakes in all capital cities on mainland Australia [16]. The distribution of tiger snakes in South Australia is generally limited to the Adelaide Hills area [17] and along the Murray River [16], which is consistent with the findings in the present study. A statistically significant conclusion reached in this study was the determination that envenomated dogs and cats were $20 \%$ less likely to survive the ordeal when diagnosis of snake species was made by means other than the use of a SVDK. This is likely due to the fact that clinical signs alone are an unreliable indicator of snake species, due to the significant overlap of the reported signs $[7,15]$. This finding was unexpected given the fact that the majority of cases being presented to veterinary clinics were suspected or proven to be a result of brown snake envenomation. Also, study by Best [3] reported no statistical significance in dog survival when offending snake species was known, with 92\% survival with known species, and 93\% survival with unknown species. There was however a significance in cats, with $92 \%$ survival when snake species was known, and $82 \%$ survival 
when unknown. Moreover, it is worth to note that a dry bite, snakebite with non-envenomation, may also occur, which could contribute to negative SVDK results due to no envenomation. Dry bite from eastern brown snake has been reported to occur at relatively high rate, up to $80 \%$ of cases in humans. In the present study, for the majority of cases of negative SVDK results, animals were brought to the two emergency clinics with obvious clinical signs of envenomation, with $78 \%$ showed recumbancy and $58 \%$ showed paresis in cats, and $36 \%$ presented with recumbancy and paresis in dogs. Thus, in our study negative SVDK results due to dry bite would be more likely for dogs than for cats, or occurred at a much lower rate in both dogs and cats, compared to that reported in humans.

It was determined that a contradictory result from the SVDK occurred approximately $14 \%$ of the time, making interpretation, diagnosis and choice of treatment for the patient more challenging. Previous studies have also reported an occurrence of contradictory results obtained from the SVDK, with an incidence of $9 \%$ to $58 \%$ negative results in cases of confirmed snake envenomation, and $4 \%$ to $11 \%$ of positive results in cases where snake envenomation was ruled out [3,11-14]. Some of the negative results obtained in cases of definite envenoming may be explained by the variation in venom levels found in blood and urine samples [9]. It has been found that if less than 8 hours have lapsed since the snakebite, then blood is the most appropriate sample, whereas urine is the most appropriate sample to obtain if the SVDK is being performed more than 8 hours since the predicted time of envenomation [9]. This indicates the importance of estimating the time elapsed since envenomation in order to allow selection of the correct sample for the SVDK. Another possible explanation is due to the phenomenon known as the hook effect, in which high venom concentrations in the sample can overwhelm the SVDK, which results in saturation of the binding antibodies. This thereby prevents correct formation of the immunoassay, leading to reduced signal strength in the target well with subsequently increased cross-reactivity in other wells $[6,18]$.

The perception that there is a high proportion of positive SVDK results in cases of nonenvenoming has been primarily attributed to operator error when performing the test [8]. A study by Ong and colleagues [8] reported that the authors tested 25 feline and 50 canine urine samples in animals admitted for reasons unrelated to snake envenomation and found no false-positive reactions occurred, proving $100 \%$ specificity of the SVDK for urine samples. Common pitfalls that have been associated with use of a SVDK include inappropriate storage of kits, using out-of-date kits, using an inappropriate sample based on time since envenomation, failing to adequately wash the test strip after the first incubation phase, using paper towel to blot the wells resulting in introduction of loose fibres, and failure to continuously observe the kit during the test to determine which well turns blue first [19]. A feasible recommendation to reduce the incidence of inconclusive results would be to provide in-house training for the standardisation of technique when using the SVDK.

Generally, a sample taken from the bite site of an envenomated animal will provide the most accurate result, followed by urine and then blood samples [6]. As bite sites in envenomated animals were rarely found, this might be another possible explanation for invalid SVDK results being encountered. Bite sites are however notorious for being difficult to locate in veterinary patients, which is likely due to their hair coat $[15,16,20]$. A display of pre-paralytic signs of envenomation, such as temporary collapse, salivation and lethargy followed by spontaneous recovery, indicates that a potentially lethal envenomation has occurred [3]. This was seen in $22 \%$ of dogs, and was due to low molecular weight substances in the venom that diffuse rapidly from the bite site [3]. Recovery is however followed by gradual deterioration as other venom components begin interacting with the body. This phenomenon is seen often in dogs, but rarely seen in cats, where the earliest signs are usually ataxia and weakness [3].

Common neurotoxic signs associated with both brown and tiger snake envenomations in this study were recumbancy, ataxia due to hind limb paresis, dyspnoea, and ocular neuropathy. These signs of neurotoxicity correlate well with findings of previous studies reported in dogs $[7,20,21]$ and cats $[9,21,22]$. The presence of these non-specific neurological signs can be explained by the presence of neurotoxins in the venoms of all snake species, with all lethal envenomations ultimately ending in flaccid paralysis [3]. The presence of myolysins and cytotoxins is unique to tiger snake venom. In dogs, these myotoxic substances affect primarily skeletal muscle [3], with the severity of damage 
related to the amount of venom injected [23]. The resultant myonecrosis causes an increase in serum creatinine kinase (CK) levels, which has been shown to remain elevated for up to 48 hours [24]. In the 3 cases of tiger snake envenomation in the present study, measurements of CK were either not performed or not documented in the clinical notes. There was, however, elevated serum CK levels detected in 3 dogs and 4 cats with suspected or confirmed brown snake envenomation. Brown snake venoms do not contain any myotoxic agents, thus it is unclear why this occurred.

Coagulopathy can be explained by the presence of venom components in both brown and tiger snakes, namely procoagulant prothrombin activator. This condition prevents the blood from clotting due to inappropriate activation and consumption of the coagulation factors [3]. The animal is then predisposed to undergo excessive or prolonged bleeding, which occurred spontaneously or followed an injury or medical procedure. Only $17 \%$ of dogs and $7 \%$ of cats in the present study were noted to have evidence of clinical bleeding, which presented as haemorrhagic diarrhoea, or bleeding at the venepuncture or bite site. This is similar to previously reported clinical signs of coagulopathy in both human and veterinary patients $[14,15,25,26]$. However, in contrast to other studies in which all patients with clinical bleeding survived $[14,15,25]$, it was seen to be a negative prognostic indicator in this study, with $33 \%(2 / 6)$ of dogs and $60 \%(3 / 5)$ of cats died or being euthanased due to suspected cerebral haemorrhage. Taking into account that coagulation tests were not performed routinely for all animals, out of those tested in this study, $73 \%$ of dogs had prolonged PT, and $67 \%$ of dogs had prolonged aPTT, compared to cats that had prolonged PT $57 \%$ of the time, and prolonged aPTT $48 \%$ of the time. Thus laboratory evidence of coagulopathy was more common in dogs than cats. Previous studies have reported similar findings $[15,26]$, which was thought to be attributed to the fact that cats are often presented to veterinary clinics long after being bitten, which allows time for the coagulation factors to regenerate and to return towards normal [26].

Another suggested explanation as to why cats are less frequently observed to have prolonged coagulation factors is the fact that they have a higher tolerance to snake venoms [3]. In a study by Barr [21], it was reported that dogs were three times more sensitive to venom on a body-mass basis when compared with cats. Therefore, cats have an advantage of being more resistant to envenomation, which likely aids in their overall increased rate of survival. The treatment modalities employed by clinicians in the present study included the administration of antivenom and intravenous fluids, with various other combinations of supportive drugs and procedures. Previous studies have reported similar therapeutic regimes used in cases of snake envenomation $\quad[1,7,15,20-22]$. Premedication of envenomated animals with combinations of corticosteroids, antihistamines and adrenaline, was provided for $69 \%$ of dogs and $75 \%$ of cats in the present study prior to administration of the appropriate antivenom. The rationale behind the provision of premedicant agents is that common commercial antivenoms are comprised of purified portions of antibody IgG from hyperimmunised equine plasma, which have the potential to provoke hypersensitivity reactions [3]. However all the animals in the current study that suffered a hypersensitivity reaction to antivenom had been previously given premedication, which suggested that premedication did not actually reduce the occurrence of these events. Recent studies conducted in humans on the use of premedication have also reported that premedication has not been associated with a reduction in hypersensitivity reactions. Thus premedication is not currently recommended prior to the administration of antivenom in humans in Australia [27,28].

The average dose of antivenom administered to both brown and tiger snake envenomated patients in the present study was 1.75 vials for dogs, and 1 vial for cats. Previously published values for antivenom doses have ranged from a maximum of 1 vial of brown snake antivenom and 2 vials of tiger snake antivenom in both dogs and cats [20-22]. Meanwhile, in a study by Indrawirawan et al, fifteen dogs were given on average 2 vials and cats 1 vial of antivenom for both brown and tiger snake envenomation cases. Recent literature has reported that 1 vial of antivenom is adequate to treat all cases of brown snake envenomation in humans [25]. Some of these patients still had minor levels of unbound venom detected by a SVDK, but had improving clinical signs and coagulopathy, resulting in similar clinical outcomes [25]. One dose is designed to provide sufficient antivenom to neutralise the maximum venom load from 1 snake, which is unlikely to even be injected in most snakebite cases [19]. The findings of this 
study suggested that further study is required to determine the optimum dosages of antivenom required in dogs and cats [25]. Two potentially controversial treatments provided in some instances included the administration of antibiotics in $9 \%$ of cases, and fresh frozen plasma (FFP) in $2 \%$ of cases. Antibiotics were given to envenomated animals in order to prevent the occurrence of secondary bacterial infections. However secondary infections following bites from Australian snakes have been found to be extremely rare, making the use of prophylactic antibiotics inappropriate [19]. Administration of FFP for the replacement of consumed coagulation factors is also not recommended. This is because despite its ability to improve laboratory parameters, it has not been associated with improved clinical outcome in envenomated patients $[19,29]$.

Overall, the average length of hospitalisation for envenomated animals in the present study was 2.5 days for dogs and 3.1 days for cats. Previously reported hospitalisation times have been of similar length, with an average of 1.5 to 3.1 days in dogs, and 3.3 to 4.2 days in cats with all types of snake envenomation $[15,20,22]$. The overall survival rate for all cases of snake envenomation that were treated was $81 \%$ in dogs and $92 \%$ in cats. Previous reports in the literature have reported survival percentages following antivenom treatment of $75 \%$ to $95 \%$ for canine envenomation cases, and $91 \%$ to $97 \%$ for feline snake envenomation cases [1,15,20-22]. Thus, survival rates for the two South Australian Veterinary clinics involved in this study were consistent with results from previous investigations.

Acording to the manufacturer, identification of snake species in case of envenomation should not based solely on CSL SVDK, but clinical envenomation signs should be taken into account for diagnosis. In our study, in $44 \%$ of the snake envenomation cases, the diagnosis of snake identification was made without a SVDK, but based solely on clinical signs, or a combination of clinical signs and prolonged coagulation factors. For the remaining $56 \%$ of the cases, a SVDK was utilised for diagnosis. There were also considerable overlapping symptoms between brown snake and tiger snake envenomation, making accurate diagnosis more difficult to achieve. Furthermore, in case of lesser than 3 wells changed colour following another well-changed colour, this should be considered as black snake envenomation. In our study, the findings that brown snakes were responsible for $97 \%$ cases of snake envenomation and tiger snakes only $3 \%$ cases, with no other snake species identified were consistent with that reported in the literature. This is because brown snakes are reported to be the most common venomous snakes in all capital cities on mainland Australia [16]. The distribution of tiger snakes in South Australia is generally limited to the Adelaide Hills area [17] and along the Murray River [16]. This further explained why bite from other snakes, including black, death adder and taipan snakes found not found within the 155 cats and dogs studied.

A limitation encountered with the present study was the small number of animals with confirmed tiger snake envenomation, making it difficult to compare and contrast the differences between brown and tiger snake envenomation syndromes in dogs and cats. Another limitation was the inability to perform keyword searches in the Cornerstone $₫$ database. Invoices for SVDKs and antivenom sold were obtained, but this excluded the ability to search for animals with assumed or definite envenomation that died or were euthanased soon after presentation and did not receive any tests or treatment. If these animals had been included, there would have likely been reduced survival rates in the present study.

\section{CONCLUSION}

The results of the current study demonstrate that diagnosis of species-specific snake envenomation using a SVDK will lead to improved patient outcome. Care is required when interpreting results from the SVDK due to the high proportion of contradictory results. Thus it is not recommended to be used as a screening test, but rather be used as an adjunct to determine offending snake species when the animal is already showing signs of envenomation. Although only a small proportion of tiger snakebite cases were analysed in this study, it is inadvisable to diagnose snake species based on clinical signs alone due to the significant overlap between envenomation syndromes.

\section{CONSENT}

Consent was obtained from The Companion Animal Health Centre, The University of Adelaide, School of Animal and Veterinary Sciences, Roseworthy Campus, South Australia 
and The Riverland Veterinary Practice, Berri, South Australia for accessing and collecting the snakebite data/cases for this study.

\section{ETHICAL APPROVAL}

It is not applicable.

\section{ACKNOWLEDGEMENT}

The authors would like to thank The Companion Animal Health Centre, The University of Adelaide, Roseworthy Campus and The Riverland Veterinary Practice, Berri, South Australia for supporting this project.

\section{COMPETING INTERESTS}

Authors have declared that no competing interests exist.

\section{REFERENCES}

1. Mirtschin PJ, Masci P, Paton DC et al. Snake bites recorded by veterinary practices in Australia. Aust Vet J. 1998;76:195-198.

2. Department of Environment, Water and Natural Resources. Snakes.

Available:http://www.environment.sa.gov.a u/managing-natural-resources/plants-andanimals/Living with wildlife/Snakes (Accessed October 2015)

3. Best P. Snake envenomation of companion animals. In: The University of Sydney Post-Graduate Committee in Veterinary Science "Clinical Toxicology" Proceedings No 318. 1998;231-248.

4. Chippaux JP, Williams V, White J. Snake venom variability: Methods of study, results and interpretation. Toxicon. 1991;29:1279-1303.

5. Morrison JJ, Pearn J, Covacevich J, et al. Can Australians identify snakes? Med J Aust. 1983;2:66-70.

6. CSL Limited. Snake venom detection kit (SVDK) enzyme immunoassay method product leaflet. CSL Limited, Parkville, Victoria; 2007.

7. Heller J, Bosward KL, Hodgson JL, et al. Snake envenomation in dogs in New South Wales. Aust Vet J. 2005;83:286292.

8. Ong RKC, Swindells K, Mansfield CS. Prospective determination of the specificity of a commercial snake venom detection kit in urine samples from dogs and cats. Aust Vet J. 2010;88:222-224.

9. Moisidis AV, James $\mathrm{T}$, Smith $\mathrm{HV}$, et al. Snake envenomation in cats and its detection by rapid immunoassay. Aust Vet J. 1996;74:143-147.

10. Tibballs J. Diagnosis and treatment of confirmed and suspected snakebite. Implications from an analysis of 46 paediatric cases. Med J Aust. 1992;156: 270-274.

11. Sutherland SK. Antivenom use in Australia. Premedication, adverse reactions and the use of venom detection kits. Med J Aust. 1992;157:734-739.

12. Mead HJ, Jelinek GA. Suspected snakebite in children: A study of 156 patients over 10 years. Med J Aust. 1996;164:467-470.

13. Trevett AJ, Lalloo DG, Nwokolo NC, et al. Venom detection kits in the management of snakebite in central province, Papua New Guinea. Toxicon. 1995;33:703-705.

14. Isbister GK, O'Leary MA, Elliott M, Brown SGA. Tiger snake (Notechis spp) envenoming: Australian Snakebite Project (ASP- 13). Med J Aust. 2012;197:173-177.

15. Indrawirawan $\mathrm{YH}$, Sheridan $\mathrm{GI}$, McAlees TJ. Clinical features of Mainland tiger and Eastern brown snake envenomation in dogs and cats in Melbourne. Aust Vet Pract. 2014;44:704-712.

16. Shea GM. The distribution and identification of dangerously venomous Australian terrestrial snakes. Aust Vet J. 1999;77:791-798.

17. Department for Environment and Heritage. Adelaide and Mount Lofty Ranges, South Australia: Threatened Species Profile Eastern Tiger Snake; 2008.

Available:www.environment.sa.gov.au/files 1718e6fe5-bd15-49d7-ac299fdb00c2f68b/pa-fact-tigersnake.pdf (Accessed October 2005)

18. Steuten J, Winkel K, Carroll T, et al. The molecular basis of cross-reactivity in the Australian Snake Venom Detection Kit (SVDK). Toxicon. 2007;50:1041-1052.

19. White J. Snakes. In: A clinicians guide to venomous bites and stings: Incorporating the updated CSL antivenom handbook. 1st edn. bioCSL Pty Ltd, Victoria. 2013;49174. 
20. Hill FW. Snake bite in dogs. Aust Vet J. 1979;55:82-85.

21. Barr SC. Clinical features therapy and epidemiology of tiger snake bite in dogs and cats. Aust Vet J. 1984;61:208-212.

22. Hill FW, Campbell T. Snake bite in cats. Aust Vet J. 1978;54:437-439.

23. Lewis PF. Common tiger snake envenomation in dogs and micerelationship between the amount of venom injected and the onset of clinical signs. Aust Vet J. 1994;71:130-132.

24. Sharp NJ, Kornegay JN, Bartlett RJ, et al. Notexin-induced muscle injury in the dog. $\mathrm{J}$ Neurol Sci. 1993;116:73-81.

25. Allen GE, Brown SGA, Buckley NA, et al. Clinical effects and antivenom dosing in brown snake (Pseudonaja spp.) envenoming - Australian snakebite project (ASP-14). PLoS ONE. 2012;7:e53188.
26. Holloway SA, Parry BW. Observations on blood coagulation after snakebite in dogs and cats. Aust Vet J. 1989;66:364-366.

27. Isbister GK, Brown SG, MacDonald E, et al. Current use of Australian snake antivenoms and frequency of immediatetype hypersensitivity reactions and anaphylaxis. Med J Aust. 2008;188:473476.

28. Isbister GK, Brown SGA, Page CB, et al. Snakebite in Australia: A practical approach to diagnosis and treatment. Med J Aust. 2013;199:763-768.

29. Jelinek GA, Smith A, Lynch D, et al. The effect of adjunctive fresh frozen plasma administration on coagulation parameters and survival in a canine model of antivenom-treated brown snake envenoming. Anaesth Intensive Care. 2005;33:36-40.

(c) 2018 Wright and Ngo; This is an Open Access article distributed under the terms of the Creative Commons Attribution License (http://creativecommons.org/licenses/by/4.0), which permits unrestricted use, distribution, and reproduction in any medium, provided the original work is properly cited.

Peer-review history:

The peer review history for this paper can be accessed here: http://www.sciencedomain.org/review-history/24024 